\title{
Unique Mode of Lipogenic Activation in Rat Preputial Sebocytes
}

\author{
Dianne Deplewski, Kenan Qin, Nancy Ciletti, and Robert L. Rosenfield \\ Department of Pediatrics, Pritzker School of Medicine, The University of Chicago, 5841 S. Maryland Avenue (MC-5053), Chicago, \\ IL 60637-1470, USA
}

Correspondence should be addressed to Dianne Deplewski, ddeplews@peds.bsd.uchicago.edu

Received 15 January 2011; Accepted 27 May 2011

Academic Editor: Phillip B. Hylemon

Copyright ( $) 2011$ Dianne Deplewski et al. This is an open access article distributed under the Creative Commons Attribution License, which permits unrestricted use, distribution, and reproduction in any medium, provided the original work is properly cited.

\begin{abstract}
Lipoprotein delivery of fatty acids and cholesterol is linked with peroxisome proliferator-activated receptor (PPAR) activation in adipocytes and macrophages. We postulated that similar interactions exist in sebaceous epithelial cells (sebocytes) in which PPAR activation induces differentiation. High-density lipoprotein (HDL) and very low-density lipoprotein (VLDL) markedly enhanced sebocyte differentiation above that found with PPAR agonists and were more potent than explicable by their lipid content. The PPAR $\gamma$ antagonist GW5393 reduced sebocyte differentiation to all PPAR isoform agonists, HDL and VLDL, suggesting that the lipoprotein effect on differentiation occurs partially through activation of PPAR $\gamma$. Furthermore, we found that sebocytes expressed a unique pattern of lipogenic genes. Our results demonstrate that HDL and VLDL are the most potent inducers of sebocyte differentiation tested to date, and these actions are partially inhibited by PPAR antagonists. This suggests that substrates provided by lipoproteins are targeted to sebocytes and affect their own disposition via PPAR activation.
\end{abstract}

\section{Introduction}

Sebaceous epithelial cell (sebocyte) differentiation is defined by increasing accumulation of lipid droplets, the major component of sebum. Peroxisome proliferator-activated receptor (PPAR) isoforms, which were originally discovered because of their key roles in adipogenesis and lipid metabolism, have been shown to strongly stimulate sebocyte differentiation in vitro [1-4]. All PPAR isoforms have been identified in sebocytes $[1,2,5]$. PPAR $\gamma$ seems essential for sebocyte differentiation, as sebocytes do not appear to develop from cell lineages devoid of PPAR $\gamma$ [6], and PPAR $\gamma$ agonists induce lipid-droplet forming colonies (LFCs) in the primary rat preputial cell culture model of sebocyte differentiation but not in cultured epidermal cells (keratinocytes) [1]. PPAR $\delta$ has been deduced to be important in sebocyte differentiation since linoleic acid and carbaprostacyclin (cPGI2), which are agonists of both PPAR $\alpha$ and PPAR $\delta$, induce more LFCs in sebocytes than either PPAR $\alpha$ or PPAR $\gamma$ agonists [1]. Linoleic acid is the most effective stimulator of sebocyte differentiation tested thus far $[1,2]$, but its physiological relevance is suspect since its action requires a relatively high concentration $\left(10^{-4} \mathrm{M}\right)$, and it inappropriately stimulates LFCs in epidermal cells [1], which raises the possibility that it mainly acts as a fatty acid substrate for lipogenesis.

Recently, PPAR action in macrophages and adipocytes has been shown to be intimately linked with lipoprotein delivery of fatty acids and cholesterol [7-14]. The relationship is complex, and the actions of PPAR isoforms may be interactive. For example, while macrophage uptake of low-density lipoprotein (LDL)-derived fatty acid metabolites is $\operatorname{PPAR} \delta$-inducible [13], it is $\operatorname{PPAR} \gamma$-dependent [7-9], yet very low-density lipoprotein (VLDL)-induced triglyceride accumulation in macrophages is absolutely PPAR $\delta$ dependent [14]. Lipoprotein is among the lipogenic regulators shown to be important for sebaceous differentiation in studies of genetically modified mice [15].

These considerations led us to test the hypothesis that lipoproteins interact with PPAR $\gamma$ and PPAR $\delta$ to induce sebocyte differentiation in a unique pattern that is related to a distinctive pattern of gene expression. Our studies are compatible with the concept that lipoproteins act to target lipid substrates within skin to sebocytes, where they induce differentiation via PPAR-mediated lipogenic pathways. 


\section{Material and Methods}

Cell Preparations and Culture. All animal experimentation was conducted in accordance with accepted standards of humane animal care. Single-cell suspensions of rat preputial sebocytes (or epidermal cells for control purposes) obtained from 60 d.o. male Sprague-Dawley rats were prepared by enzymatic digestion under sterile conditions, as previously reported [16]. Single-cell suspensions were plated on growth-arrested 3T3-J2 fibroblast feeder layers in Dulbecco's Modified Eagles Medium (DMEM) with 10\% fetal calf serum supplemented with $10^{-6} \mathrm{M}$ insulin, $10^{-10} \mathrm{M}$ choleratoxin, $10^{-6} \mathrm{M}$ cortisol, and antibiotics, as previously reported [1]. The cells were grown in primary culture in $95 \%$ air $/ 5 \%$ $\mathrm{CO}_{2}$ at $37^{\circ} \mathrm{C}$. A serum-free chemically defined cell culture medium (Cellgro Complete) was substituted for DMEM plus serum after the 3 rd day of culture. Treatments were added in triplicate for 48 hours on day 7 (preconfluence). Treatments included the PPAR activators troglitazone (Tro; specific activator of PPAR $\gamma$ ), cPGI2 (activator of PPAR $\delta, \alpha$ ), and linoleic acid (activator of PPAR $\delta, \alpha$ ). In addition, we used the specific PPAR $\delta$ agonists GW610742 (GW742) [17], and L-165041 [18], the specific PPAR $y$ agonist GW347845 (GW845) [19], the specific PPAR $\gamma$ competitive antagonist GW5393 [17] and the noncompetitive PPAR $y$ binding pocket antagonist GW9662 [20] that were kindly provided by GlaxoSmithKline and Merck, courtesy of Drs. Tim Willson and Joel Berger, respectively. Agonists and antagonists were prepared as a stock solution at $10 \mathrm{mM}$ in DMSO and stored in aliquots at $-20^{\circ} \mathrm{C}$. The antagonists were added to incubation medium 2 hours before agonists. Human lipoproteins were purchased from EMD Biosciences. For histochemical determination of sebocyte differentiation, cells were grown in $35 \mathrm{~mm} \mathrm{6-}$ well plates. For RNA preparations, Trizol reagent was either added directly to single-cell suspensions or to cultured cells attached to the plate.

Histological Analysis of Lipid Droplet Formation. LFCs were quantified by light microscopy after Oil Red O staining, as previously described [21]. A LFC is defined as a colony containing over 5 cells positive for Oil Red O staining (cells equivalent to at least middifferentiation of sebocytes), in order to clearly distinguish specific cytoplasmic staining from the amorphous staining of deteriorating cells, as previously described [1].

Triglyceride/Cholesterol Assays. Triplicate wells from selected treatments were washed in Dulbecco's phosphatebuffered saline (PBS) three times and then trypsinized, collected, and resuspended in PBS. The cell suspensions were analyzed for triglyceride and cholesterol content. A UV method for the determination of triglycerides was used according to the manufacturer (Test-Combination Glycerol, Roche). In a series of enzymatic reactions, NADPH was determined by means of its light absorbance at $340 \mathrm{~nm}$ and was directly related to the amount of glycerol or triglycerides present in the cell suspension. The cholesterol content was determined by a colorimetric method (TestCombination Cholesterol, Roche) utilizing cholesterol oxidase and reagents that generate a lutidine-dye, that is, stoichiometric to the amount of cholesterol in the sample and was measured at $405 \mathrm{~nm}$. Standard curves were generated for each experiment to determine the accuracy of the assay.

Reverse Transcriptase Polymerase Chain Reaction. RTPCR was carried out as previously described [22] with minor modifications according to the manufacturer's protocol (from SuperScript First-Strand Synthesis System for RT-PCR, Gibco BRL). Briefly, single-stranded cDNA was synthesized from $2 \mu \mathrm{g}$ total RNA using reverse transcriptase and random primers in $20 \mu \mathrm{L}$. RT-PCR for lipogenic gene expression was performed using specific primers (Table 1 ). Two $\mu \mathrm{l}$ of single-stranded cDNA in a total $50 \mu \mathrm{L}$ PCR reaction mixture was denatured for $5 \mathrm{~min}$ at $95^{\circ} \mathrm{C}$, then thirty-five cycles of PCR amplification were performed under the following conditions: $15 \mathrm{sec}$ at $94^{\circ} \mathrm{C}, 15 \mathrm{sec}$ at $50^{\circ} \mathrm{C}$, and $2 \mathrm{~min}$ at $72^{\circ} \mathrm{C}$, finally the samples were placed for $7 \mathrm{~min}$ at $72^{\circ} \mathrm{C}$. A negative control was carried out for each pair of primers with the same procedure without reverse transcriptase. Amplified DNA was resolved on a 1.5\% agarose gel containing $5 \mu \mathrm{g} / \mathrm{mL}$ ethidium bromide and bands visualized under UV light.

Statistical Analysis. One-way ANOVA followed by Fisher's Protected Least Differences post hoc test was used to compare the various treatments. Statistical analyses were performed using the Statview program; a $P$-value (two-tailed) $<0.05$ was considered statistically significant.

\section{Results}

3.1. Specific PPAR $\delta$ and PPARy Agonists Induce Sebocyte Differentiation. Recently available specific PPAR $\delta$ agonists were used to distinguish the role of PPAR $\delta$ from that of PPAR $\alpha$ in sebocyte differentiation. We show that the specific PPAR $\delta$ agonist (GW742) exhibits a dose-response effect on sebocyte differentiation $(P<0.001$; Figure 1$)$ and is equipotent to the PPAR $\delta, \alpha$ agonist cPGI2 at $1 \mu \mathrm{M}$. A similar statistically significant dose-response effect on sebocyte differentiation was found with the specific PPAR $\delta$ agonist L-165041 (data not shown). These results support a specific role of PPAR $\delta$ in sebocyte lipogenesis.

The specific PPAR $y$ agonist (GW845) also exhibited a dose-related effect on sebocyte differentiation $(P<0.01$; Figure 1). Furthermore, it was the most potent of all PPAR isoform agonists tested. $(P<0.01$; Figure 1$)$. These results add further evidence of the importance of PPAR $\gamma$ activation on sebocyte differentiation.

3.2. PPARy Antagonists Block PPARy and PPAR $\delta$ Action in Sebocytes. The specific PPAR $y$ competitive antagonist GW5393 at $1 \mu \mathrm{M}$ specifically inhibited the response to all PPAR $\gamma$ agonists: the LFC responses to the lowest doses of the potent specific PPAR $\gamma$ agonist (GW845) and the low potency troglitazone were completely blocked, and those to higher doses of GW845 were significantly inhibited (Figure 1(a)). As expected, LFC formation in response to the lowest dose of the specific PPAR $\delta$ agonist GW742 was not significantly inhibited by GW5393. However, the effect of higher doses of the PPAR $\delta$ agonist and of the nonspecific PPAR $\delta, \alpha$ agonists cPGI2 and linoleic acid were partly inhibited by GW5393. We interpret this as indicating that low-level PPAR $\delta$ stimulation 


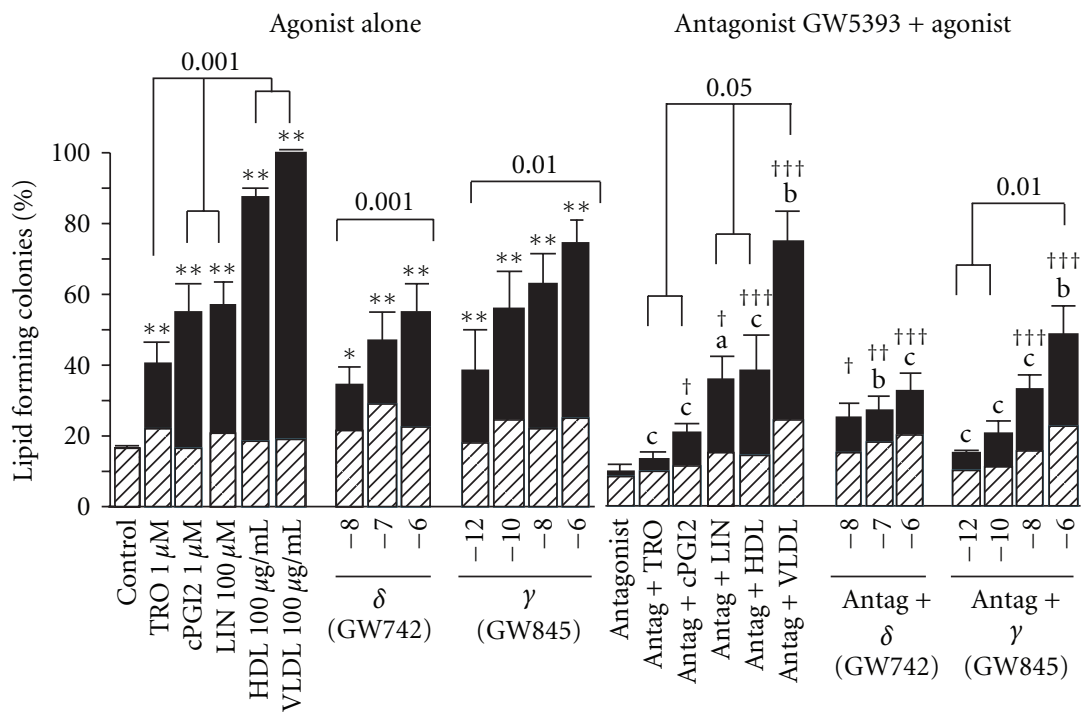

\begin{tabular}{|c|c|}
\hline — ORO-POS. >50 & $P$ versus antagonist alone \\
\hline ORO-POS. 6-50 & ${ }^{\dagger} P<0.05$ \\
\hline$P$ versus control & ${ }^{\dagger \dagger} P<0.01$ \\
\hline$* P<0.01$ & $\dagger^{\dagger \dagger} P<0.001$ \\
\hline$* * P<0.001$ & \\
\hline
\end{tabular}

(a)

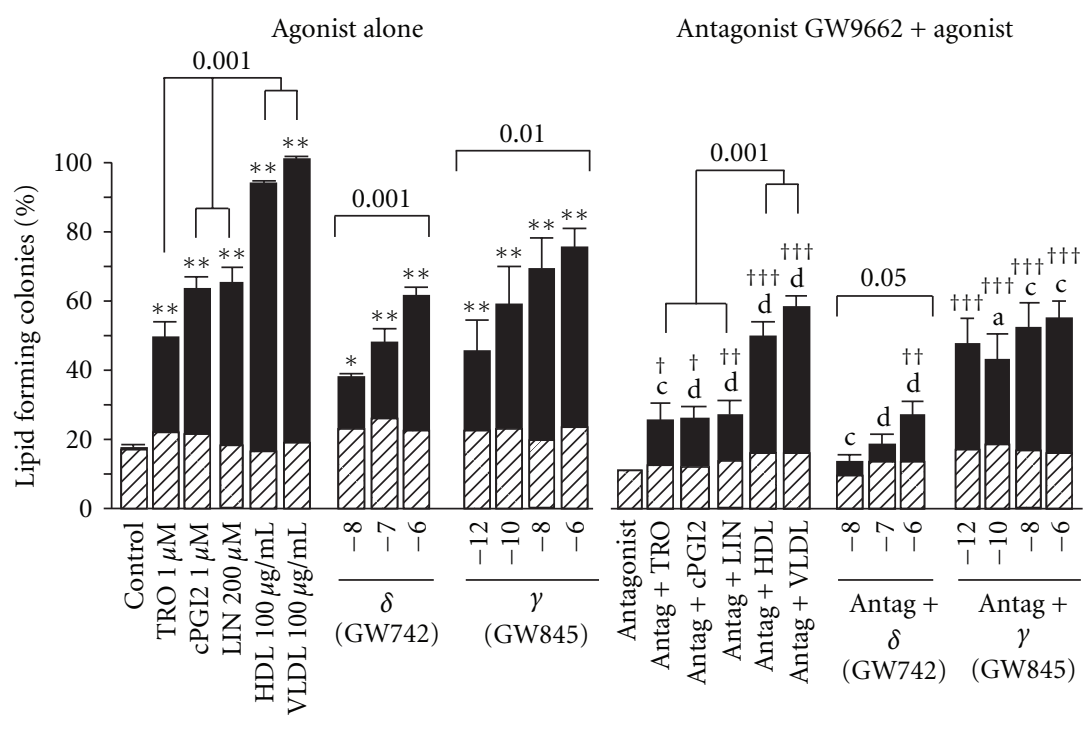
(a) $P<0.02$
(b) $P<0.01$
(c) $P<0.001$
$n=4-5$

$P$ versus same agonist alone

$\frac{P \text { versus antagonist alone }}{}$
${ }^{\dagger} P<0.05$
${ }^{\dagger \dagger} P<0.02$
${ }^{\dagger \dagger} P<0.001$

$P$ versus same agonist alone
(a) $P<0.05$
(b) $P<0.02$
(c) $P<0.01$
(d) $P<0.001$

(b)

FIGURE 1: Comparison of sebocyte differentiation induced by PPAR agonists and lipoproteins before and after pretreatment with two PPAR antagonists. Maximally effective doses of the selective PPAR agonists troglitazone (TRO), carbaprostacyclin (cPGI2), and linoleic acid (LIN) were used as indicated. HDL and LDL were used at $100 \mu \mathrm{g}$ protein/mL. The specific PPAR $\delta$ (GW742) and PPAR $\gamma($ GW845) agonists were used at the doses indicated. The PPAR $\gamma$ antagonist GW5393 (a) and the PPAR binding pocket antagonist GW9662 (b) were added to the cells at a dose of $1 \mu \mathrm{g} 2$ hours prior to treatment with the PPAR agonists or lipoproteins on day 7 of primary culture. LFC determination was made on day 9 of culture after fixing and staining the cells with Oil Red O (ORO). Striped bars indicate colonies with 6-50 ORO-stained cells, and solid bars those colonies with >50 ORO-stained cells. Means +/- SEMs are presented. 
TABLE 1: Primers for RT-PCR.

\begin{tabular}{|c|c|c|}
\hline Primer & $\begin{array}{l}\text { Primer sequence } \\
\text { Forward }\left(5^{\prime} \text { to } 3^{\prime}\right)\end{array}$ & Reverse $\left(5^{\prime}-3^{\prime}\right)$ \\
\hline ACAT-1 & 5'-ATGGCTGCCCTGGCGGTTCTA-3' & 5'-CTACAGCTTCTCAATCAGCAC-3' \\
\hline Add-1 & 5'-ATGGATTGCACATTTGAAGAC-3' & 5'-TGGTACTGTGGCCAGGATGGT-3' \\
\hline Adipsin & 5'-ATGCACAGCTCCGTGTACCTC-3' & 5'-TCAGGCCGTCACGTTAACACT-3' \\
\hline aP2 & 5'-TGGAAACTCGTCTCCAGTGAG-3' & $5^{\prime}$-CAAATTTCAGTCCAGGGCCTC-3' \\
\hline Apo-E & 5'-ATGAAGGCTCTGTGGGCCCTG-3' & $5^{\prime}$-TCATTGATTTCTCCAGGGCAC-3' \\
\hline CD36 & 5'-GCAGCTGCACCACATATCTAC-3' & 5'-GCTGGCTTGACCAGTATGTTG-3' \\
\hline DGAT-1 & 5'-GCGGTTTCAGCAATTACCGTG-3' & 5'-ACTGGGGCATCATAGTTGAGC-3' \\
\hline HSL & 5'GAAACCTAGGAGACCAATTTC-3' & 5' -ACCTGCAAAGACGTTGGACAG-3' \\
\hline Leptin & 5'-ATGACATTTCACACACGCAGT-3' & $5^{\prime}$-CTAGAGGAGTAGGAGAAACGG -3' \\
\hline Leptin-R & 5'-ATGACGTGTCAGAAATTCTATG-3' & 5'-GGAAGCATTGGATCCAACACT-3' \\
\hline LPL & 5'-ATGGAGAGCAAAGCCCTGCTC-3' & 5'-CAGAGACTTGTCATGGCATTT-3' \\
\hline MC5-R & $5^{\prime}$ - ATGAACTCCTCGTCTCACCTG -3' & $5^{\prime}$ - TTAATACCTGCCAAGGAGCGT -3' \\
\hline Scd-1 & 5'-ATGCCGGCCCACATGCTCCAA-3' & 5'-TCAGCTACTCTTGTGGCTCCC-3' \\
\hline SR-B1 & 5'-ATCATGATTCTCATGGTGCCC-3' & $5^{\prime}$-TGGCAGCTGGTGACATCAGAG-3' \\
\hline
\end{tabular}

acyl-CoA:cholesterol acyltransferase type 1 (ACAT-1), adipocyte determination factor (Add-1), adipocyte fatty acid binding protein (aP2), apolipoprotein E (ApoE), fatty acid translocase (CD-36), acyl-CoA:diacylglycerol acyltransferase type 1 (DGAT1), hormone sensitive lipase (HSL), leptin receptor (Leptin-R), lipoprotein lipase (LPL), melanocortin 5 receptor (MC5-R), stearoyl-CoA desaturase type 1 (Scd-1), and scavenger receptor type BI (SR-B1).

induces sebocyte lipogenesis and that higher level PPAR $\delta$ stimulation of lipogenesis involves signaling via PPAR $\gamma$.

The PPAR $\gamma$ binding pocket antagonist GW9662 completely blocked the effect of the specific PPAR $\delta$ agonist GW742 at the lower doses and did not inhibit the effect of the specific PPAR $\gamma$ agonist GW845 at the lowest dose (Figure 1(b)). Although GW9662 behaves as a relatively specific PPAR $\gamma$ antagonist in adipocytes [20], these studies indicate that GW9662 acts functionally as a specific inhibitor of PPAR $\delta$ in sebocytes. This suggests that the apparent cellspecific effects of GW9662 are dependent on cell-specific expression of coregulator molecules. GW9662 inhibited the responses to higher doses of both these agonists and to all other PPAR activators. These data support the concept of cooperation in the actions of PPAR $\delta$ and PPAR $\gamma$ in sebocyte lipogenesis.

3.3. The Lipoproteins HDL and VLDL Are Potent Inducers of Sebocyte Differentiation. Since lipoproteins deliver cholesterol and fatty acids to cells, we tested the effect of lipoproteins on sebocyte differentiation. HDL $100 \mu \mathrm{g}$ protein/mL stimulated significantly more sebocyte LFCs ( $89 \pm$ 3\% SEM; $P<0.001$ ) than any PPAR agonist (Figure 1). VLDL $100 \mu \mathrm{g}$ protein/mL tended to induce even more LFCs $(99 \pm 1 \%)$. In addition, sebocytes exhibited distinct patterns of cholesterol and triglyceride accumulation following treatment with HDL and VLDL (Figure 2). HDL stimulated predominantly cholesterol accumulation, along with a lesser, but significant, triglyceride accumulation. VLDL stimulated primarily triglyceride accumulation. These patterns of lipid accumulation reflect the distinct core lipid composition of these two lipoproteins [23]: HDL $100 \mu \mathrm{g}$ protein/ml contains approximately $100 \mu \mathrm{g}$ lipid/mL, of which the core lipid constitutes about half and consists of approximately $78 \%$ cholesteryl ester and 22\% triglyceride, while VLDL $100 \mu \mathrm{g}$ protein/mL contains approximately $1000 \mu \mathrm{g}$ lipid/mL, of

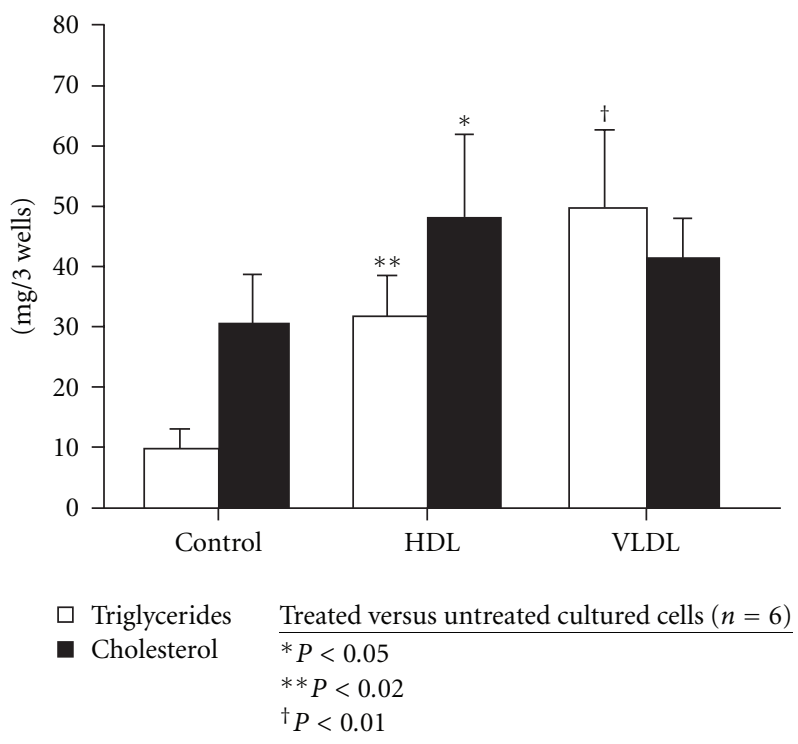

FIGURE 2: Triglyceride and cholesterol content of cultured sebocytes following treatment with HDL and VLDL. HDL induced significantly greater concentrations of both triglyceride and cholesterol, whereas VLDL induced a high level of triglyceride accumulation, but had no significant effect on cholesterol accumulation. Triglyceride $(n=5)$ and cholesterol $(n=6)$ were assayed per 3 wells. Means +/- SEMs are shown.

which the core lipid constitutes about three-quarters and consists of approximately $18 \%$ cholesteryl ester and $82 \%$ triglycerides. Thus, HDL and VLDL appear to act in part by delivering substrate for lipogenesis. However, this is probably receptor mediated for reasons discussed below.

3.4. Evidence that the Apoprotein Component of HDL and VLDL Plays a Role in Sebocyte Differentiation. VLDL had a dose-response effect on sebocyte differentiation over the 


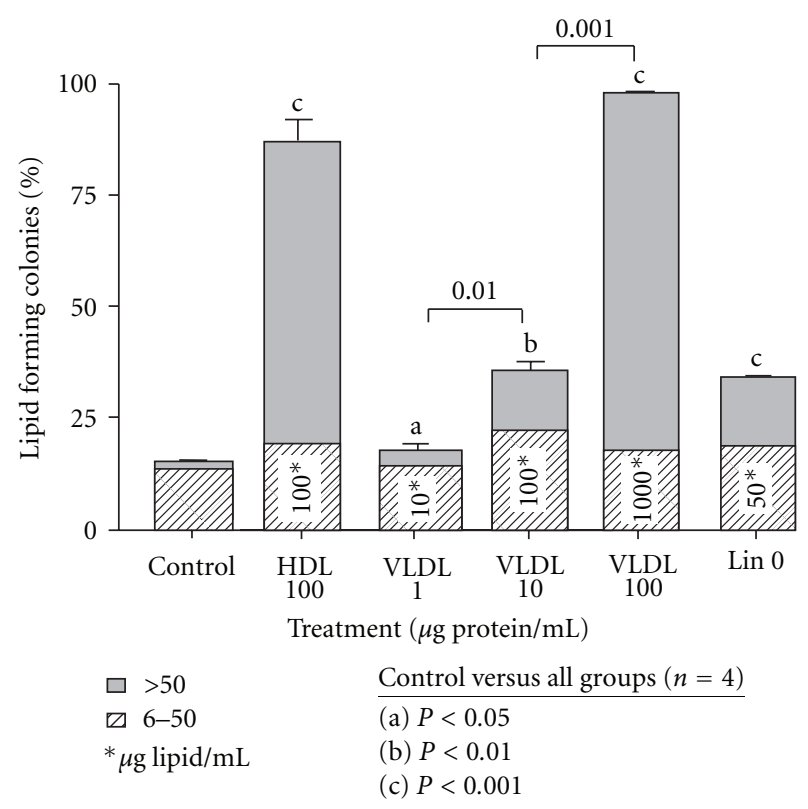

Figure 3: Comparison of protein versus lipid content of HDL, VLDL, and LIN on induction of sebocyte differentiation. VLDL was more potent than HDL in the induction of sebocyte differentiation in terms of protein content but less potent when comparing lipid content $(n=4)$. Means $+/$ - SEMs are shown.

range $1-100 \mu \mathrm{g}$ protein/mL (Figure 3 ). VLDL was as effective in inducing LFCs as HDL at a comparable apoprotein dosage of $100 \mu \mathrm{g}$ protein/mL but was less effective at an equivalent lipid concentration $(100 \mu \mathrm{g} \operatorname{lipid} / \mathrm{mL})$.

HDL was more effective than linoleic acid at a dose that had about $8 \%$ triglyceride content. Despite using submaximal doses as low as $25 \mu \mathrm{g}$ protein/mL (about $25 \mu \mathrm{g}$ lipid/mL), HDL was significantly more potent than linoleic acid, $200 \mu \mathrm{g} / \mathrm{ml}(100 \mu \mathrm{M}$; data not shown). VLDL was as effective in inducing LFCs as a dose of linoleic acid that delivers approximately half the amount of fatty acid (Figure 3). Notably, unlike linoleic acid, neither lipoprotein significantly stimulated LFCs in keratinocytes, suggesting a sebocyte-specific effect (data not shown). In addition, human LDL had no significant effect on sebocyte differentiation in our sebocyte culture system (data not shown). These considerations suggest that the apoprotein component of lipoproteins plays a key role in sebocyte differentiation and that the mechanism of lipoprotein action is not simply due to passive delivery of lipid substrate.

3.5. PPAR $\delta$ and PPARy Antagonists Attenuate Lipoprotein Effects on Sebocyte Differentiation. Both PPAR $y$ antagonists significantly inhibited LFC formation in response to HDL or VLDL (Figure 1). This suggests that not only do HDL and VLDL deliver lipid substrate to sebocytes, but also PPARs partially mediate the effects of these lipoproteins on induction of sebocyte differentiation. This conclusion is supported by the finding that HDL and VLDL effects were not discernibly augmented by PPAR agonists (data not shown), which is consistent with action via common pathways. The data are compatible with the concept that
HDL and VLDL supply one or more critical signaling molecules that act downstream of PPAR $\gamma$ action, perhaps through the same PPAR $\gamma$-independent pathways stimulated by $\operatorname{PPAR} \delta$.

3.6. Sebocytes Express a Unique Pattern of Lipogenic Genes. The role of PPARs and lipoproteins in sebocyte differentiation suggests that the mode of molecular genetic regulation of lipogenesis in sebocytes may be different in some respects from that in fat cells. Lipogenic gene expression was therefore explored by RT-PCR (Figure 4). Sebocytes were found to have a unique molecular fingerprint of lipogenic gene expression. Adipsin, adipocyte fatty acid binding protein (aP2), fatty acid translocase (CD-36), and melanocortin 5 receptor (MC5-R) were only detected in sebocytes, not in epidermal cells. Leptin was detected in cultured sebocytes (which are immature) to a greater extent than in freshly dispersed sebocytes (which are predominantly mature) or epidermal cells. The higher leptin gene expression in cultured than in freshly dispersed cells suggests that leptin expression may be upregulated early in differentiation then downregulated in late-differentiated sebocytes. In contrast, MC5-R was detected in freshly dispersed sebocytes but not in cultured sebocytes. MC5-R thus appears to be a marker of a later stage of differentiation than is achieved in culture since it is found only in freshly dispersed sebocytes. Other lipogenic genes including acyl-CoA:cholesterol acyltransferase type 1 (ACAT-1), adipocyte determination factor (Add-1), apolipoprotein E (ApoE), acyl-CoA:diacylglycerol acyltransferase type 1 (DGAT1), leptin receptor, lipoprotein lipase (LPL), stearoyl-CoA desaturase type 1 (Scd-1), and scavenger receptor type BI (SR-B1) were expressed in both sebaceous cells and epidermal cells at all stages of maturation. Androgen (dihydrotestosterone) had no clear effect on expression of any gene tested.

\section{Discussion}

Our studies show that the lipoproteins HDL and VLDL are the most effective inducers yet reported of rat sebocyte colony differentiation. Our results also indicate that PPAR $\delta$ plays a unique role in sebocyte differentiation. Notably, HDL, VLDL, and PPAR $\delta$ all boost lipogenesis in a seemingly $\operatorname{PPAR} \gamma$-dependent manner. Our data are consistent with a model in which lipoproteins specifically target lipids within skin to sebocytes, where these lipids serve a dual role, acting both as substrate and to generate signaling molecules that induce differentiation via cell-specific PPAR-mediated lipogenic pathways (Figure 5).

Lipoproteins interact with cells by binding of their apoprotein to receptors, allowing for transport of their constituent lipids into cells where they provide lipids both as substrates for intracellular metabolism and as signaling ligands for PPARs. Thus, regardless of their intracellular function, their initial interaction involves association with cell surface receptors, which could discriminate among particular classes of lipoproteins. The potential importance of this receptor interaction is suggested by the selectivity of HDL and VLDL for sebocyte differentiation in comparison 


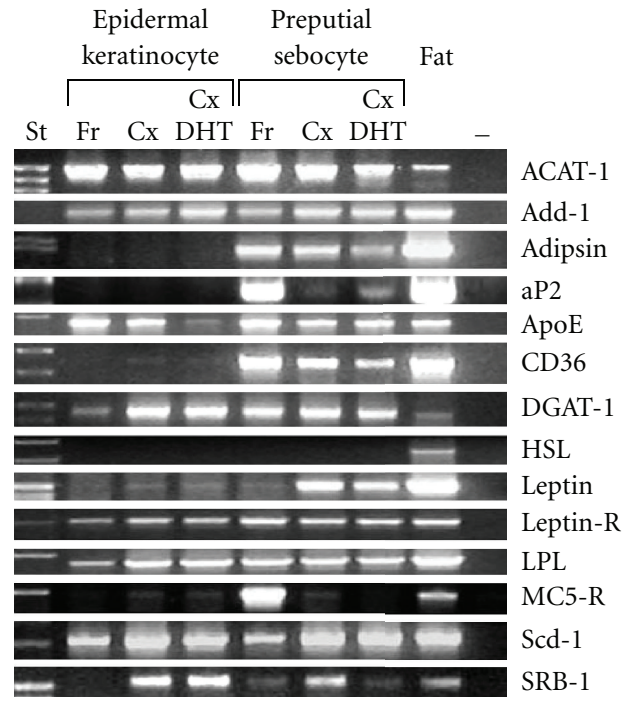

FIgURE 4: Comparison of the expression of lipogenic regulatory genes in freshly dispersed (Fr) and cultured (Cx) \pm dihydrotestosterone (DHT) preputial sebocytes and epidermal cells, using RTPCR. Total RNA was isolated from adult rat preputial sebocytes, epidermal cells and homogenized epididymal fat pad. Primers specific for the listed genes were used (see Table 1). Adipsin, aP2, CD36, and MC5-R were only detected in sebocytes, not in fresh or cultured epidermal cells. Leptin was detected in cultured sebocytes (less mature) to a greater extent than in freshly dispersed sebocytes (more mature) or epidermal cells. MC5-R was detected in freshly dispersed sebocytes to a greater extent than cultured sebocytes.

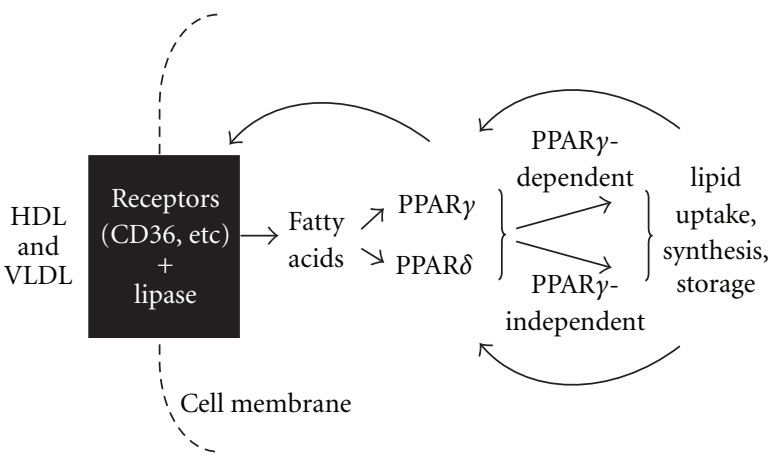

Figure 5: Feed-forward model of sebocyte differentiation. We postulate that lipoproteins target lipid substrates within skin to sebocytes through interactions with specific receptors. The substrates in turn stimulate sebocyte differentiation by acting through cell-specific PPAR $\gamma$-mediated lipogenic pathways.

to linoleic acid, which also acts as both a lipid substrate and a PPAR agonist, yet indiscriminately induces lipid droplet formation in both sebocytes and keratinocytes [1]. Our data suggest that the effectiveness of the lipoproteins is due to a regulatory effect of the apoprotein moiety rather than the simple delivery of substrates. The importance of receptor interaction is further supported by the finding that VLDL is at least, if not more potent than HDL when matched for protein concentration, but less potent when presented at similar lipid concentrations. The lack of induction of
TABLE 2: Comparison of effects of PPAR agonists and lipoproteins on lipid storage in adipocytes, macrophages, and sebocytes.

\begin{tabular}{lccc}
\hline Substrates & Adipocyte & Macrophage & Sebocyte \\
\hline PPAR $\gamma$ & positive & negative & positive \\
PPAR $\delta$ & positive/negative & positive & positive \\
LDL & $?$ & positive (oxLDL) & no effect \\
HDL & $?$ & $?$ & positive \\
VLDL & positive & $?$ & positive \\
\hline
\end{tabular}

oxLDL: oxidized low-density lipoprotein.

sebocyte differentiation by human LDL militates against the possibility of lipoproteins passively transferring lipid to target cells. Paradoxically, this finding is compatible with a role for the LDLR in mediating lipoprotein action, since the rodent LDL receptor has a much lower affinity for human LDL than it has for rodent LDL [24].

The role of PPAR $\delta$ in sebocyte differentiation is unique. Not only is PPAR $\delta$ the predominant PPAR isoform in sebocytes [25], but the specific PPAR $\delta$ agonists GW742 and L-165041 also are as potent as the previously described PPAR $\delta, \alpha$ agonist cPGI2 and more potent than 100-fold higher concentrations of linoleic acid. Furthermore, our data support the concept that $\operatorname{PPAR} \delta$ stimulates lipogenesis via both PPAR $\gamma$-dependent and-independent pathways. We found that the modest effect of a low dose of the specific PPAR $\delta$ agonist GW742 could not be blocked by the specific PPAR $y$ antagonist GW5393 that blocked the more marked effect of high dose GW742 stimulation. This suggests that high-dose PPAR $\delta$ stimulation activates PPAR $y$ signaling pathways like those involved in linoleic acid action: linoleic acid, a direct PPAR $\delta, \alpha$ agonist, functions as an indirect $\operatorname{PPAR} \gamma$ agonist in adipocytes [26] and in macrophages via its oxidized metabolites 9- and 13-HODE [7].

Although PPAR $\delta$ appears to be important in sebocyte differentiation, the specific PPAR $\gamma$ agonist GW845 and HDL and VLDL are more effective inducers of sebocyte differentiation. PPAR $\gamma$ signaling seems critical for the action of both lipoproteins and all other PPAR isoform agonists, since the specific PPAR $y$ competitive antagonist GW5393 inhibits the effects of all substantially.

The pattern of PPAR effects in sebocytes differs from that in other model systems (Table 2). The PPAR $y$ agonist stimulation of sebocyte lipid metabolism resembles that in fat cell (adipocyte) differentiation but is opposite to the effects on macrophages, in which PPAR $\gamma$ promotes lipid efflux rather than lipid storage $[13,27,28]$. On the other hand, PPAR $\delta$ agonist stimulation of sebocyte lipid formation is similar to that found in the macrophage, where PPAR $\delta$ agonist induces storage of lipids derived from oxLDL and serum [13], and contrasts to its action in adipocytes where it has equivocal effects on lipid storage and the overall net effect of promoting lipid catabolism [29].

Our studies also demonstrate that sebocytes have a characteristic molecular fingerprint: unlike keratinocytes, they expressed adipsin, aP2, CD36, and MC5-R, and immature sebocytes strongly expressed leptin, all of which play important roles in lipogenesis. The unique expression pattern of 
CD-36 makes it a candidate receptor to explain the specificity of HDL for promoting sebocyte lipogenesis in skin. CD-36 acts as a lipoprotein receptor and mediates the transport of long-chain fatty acids in mature adipocytes, macrophages, and other tissues following HDL binding [12, 30-32]. CD36 expression has also recently been demonstrated on the surface of SZ95 human sebocytes where it is thought to play a role in the transport of free fatty acids [33]. The scavenger receptor SR-B1 acts similarly, but its skin expression pattern is not confined to sebocytes; thus it would not seem to be a candidate for mediating the sebocyte-specific action of lipoproteins.

This molecular fingerprint further suggests that expression of a unique pattern of lipogenic genes underlies sebocyte differentiation. Adipsin is used by adipocytes to determine their rate of glycerol incorporation into fatty acids [34]. aP2 is an intracellular fatty acid transport protein that appears to play a role in intracytoplasmic fatty acid trafficking $[35,36]$ and is induced by PPARs [13]. MC5-R, an appetite suppressive hormone involved in the regulation of adipose stores, has been identified in preputial and human sebaceous glands $[37,38]$, where it mediates the augmentation of androgen action by melanocyte stimulating hormone- $\alpha[39,40]$. Disruption of the MC5-R gene in mice results in hypoplasia of sebaceous and preputial glands with decreased sebum production [41]. Leptin is a hormone secreted by adipocytes that is a key metabolic satiety signal in hypothalamic appetite control [42] and may downregulate fatty acid desaturation [43]. MC5-R and leptin seem to be expressed at distinctly different time points during sebocyte differentiation. MC5$\mathrm{R}$ mRNA is expressed primarily in the highly differentiated sebocytes of freshly dispersed sebocytes, but little if at all in cultured (immature) sebocytes or epidermal cells. In contrast, we found leptin mRNA to be expressed in immature sebocytes, but it was consistently barely detectable in mature sebocytes and cultured and freshly dispersed epidermal cells. Remarkably, with the exception of MC5-R, these genes are not among the lipogenic genes previously identified as essential for sebogenesis, all of which are expressed in keratinocytes as well as sebocytes: ACAT-1 [44, 45], DGAT-1 [46], and Scd-1 [47, 48].

Interestingly, addition of androgen (dihydrotestosterone) to our culture system had no clear effect on expression of any gene tested. Whereas sebaceous gland growth has been shown to be dependent on androgen in vivo, androgens alone have not been shown to have a clear effect on sebocyte differentiation in vitro [2, 4, 49]. Androgens, however, have been shown to enhance the effect of PPAR agonists on sebocyte differentiation. Nevertheless, there is no convincing evidence that this suffices to bring about complete maturation of sebaceous cell differentiation in vitro. Consequently, the mechanism by which androgens bring about full sebaceous gland development is not yet fully understood.

\section{Conclusions}

On the basis of these studies, we propose a feed-forward mechanism of sebocyte differentiation that operates through
$\operatorname{PPAR} \gamma$-dependent and $\operatorname{PPAR} \gamma$-independent pathways (Figure 5). HDL and VLDL are the most potent inducers of sebocyte differentiation and are both more effective than explicable by their lipid content, suggesting that their apoproteins moieties mediate their effects. In addition, HDL and VLDL are selective for the induction of sebocyte differentiation, as compared to keratinocyte differentiation. These observations suggest the involvement of specific lipoprotein receptors, such as CD-36, in activating signaling pathways in a hierarchy that is specific for sebocytes. In turn, accumulating lipids, such as linoleic acid itself, seem to act as both substrates and agonists of PPAR $\delta$ and PPAR $\gamma$ to amplify multiple lipid metabolic pathways for net lipid uptake and storage including further PPAR $\gamma$-dependent stimulation of CD36 expression [7-9, 13].

There is still much unknown about sebocyte differentiation. Elucidation of the mechanism of sebocyte lipogenesis will require a further understanding of the coordinated function of the many requisite genes and gene products.

\author{
Abbreviations \\ ACAT-1: acyl-CoA: cholesterol acyltransferase type 1 \\ Add-1: adipocyte differentiation and determination \\ factor-1 \\ aP2: $\quad$ adipocyte fatty acid binding protein \\ ApoE: apolipoprotein E \\ DGAT1: acyl-CoA: diacylglycerol acyltransferase type 1 \\ cPGI2: carbaprostacyclin \\ CD-36: fatty acid translocase \\ HDL: $\quad$ high density lipoprotein \\ HSL: hormone sensitive lipase \\ Leptin-R: leptin receptor \\ LFCs: lipid-droplet forming colonies \\ LPL: lipoprotein lipase \\ LDL: low-density lipoprotein \\ MC5-R: melanocortin 5 receptor \\ PPAR: peroxisome proliferator- activated receptor \\ Scd-1: $\quad$ stearoyl-CoA desaturase type 1 \\ SR-B1: scavenger receptor type BI \\ Tro: troglitazone \\ VLDL: and very low-density lipoprotein.
}

\section{Acknowledgments}

These studies were supported by the NIH (RLR-R01-HD06308, R01-HD-39267) and Louis Block Fund (DD). We thank Godfrey Getz, M.D., Ph.D, for his helpful advice.

\section{References}

[1] R. L. Rosenfield, A. Kentsis, D. Deplewski, and N. Ciletti, "Rat preputial sebocyte differentiation involves peroxisome proliferator-activated receptors," Journal of Investigative Dermatology, vol. 112, no. 2, pp. 226-232, 1999.

[2] W. Chen, C. C. Yang, H. M. Sheu, H. Seltmann, and C. C. Zouboulis, "Expression of peroxisome proliferator-activated receptor and CCAAT/enhancer binding protein transcription 
factors in cultured human sebocytes," Journal of Investigative Dermatology, vol. 121, no. 3, pp. 441-447, 2003.

[3] N. Akimoto, T. Sato, C. Iwata et al., "Expression of perilipin A on the surface of lipid droplets increases along with the differentiation of hamster sebocytes in vivo and in vitro," Journal of Investigative Dermatology, vol. 124, no. 6, pp. 11271133, 2005.

[4] E. Makrantonaki and C. C. Zouboulis, "Testosterone metabolism to $5 \alpha$-dihydrotestosterone and synthesis of sebaceous lipids is regulated by the peroxisome proliferator-activated receptor ligand linoleic acid in human sebocytes," British Journal of Dermatology, vol. 156, no. 3, pp. 428-432, 2007.

[5] M. M. Downie, D. A. Sanders, L. M. Maier, D. M. Stock, and T. Kealey, "Peroxisome proliferator-activated receptor and farnesoid X receptor ligands differentially regulate sebaceous differentiation in human sebaceous gland organ cultures in vitro," British Journal of Dermatology, vol. 151, no. 4, pp. 766775, 2004.

[6] E. D. Rosen, P. Sarraf, A. E. Troy et al., "PPAR gamma is required for the differentiation of adipose tissue in vivo and in vitro," Molecular Cell, vol. 4, no. 4, pp. 611-617, 1999.

[7] L. Nagy, P. Tontonoz, J. G. Alvarez, H. Chen, and R. M. Evans, "Oxidized LDL regulates macrophage gene expression through ligand activation of PPAR $\gamma$," Cell, vol. 93, no. 2, pp. 229-240, 1998.

[8] A. Chawla, Y. Barak, L. Nagy, D. Liao, P. Tontonoz, and R. M. Evans, "PPAR-gamma dependent and independent effects on macrophage-gene expression in lipid metabolism and inflammation," Nature Medicine, vol. 7, no. 1, pp. 48-52, 2001.

[9] K. J. Moore, E. D. Rosen, M. L. Fitzgerald et al., "The role of PPAR-gamma in macrophage differentiation and cholesterol uptake," Nature Medicine, vol. 7, no. 1, pp. 41-47, 2001.

[10] C. H. Lee, P. Olson, and R. M. Evans, "Minireview: lipid metabolism, metabolic diseases, and peroxisome proliferatoractivated receptors," Endocrinology, vol. 144, no. 6, pp. 22012207, 2003.

[11] G. Chinetti, F. G. Gbaguidi, S. Griglio et al., "CLA-1/SR-BI is expressed in atherosclerotic lesion macrophages and regulated by activators of peroxisome proliferator-activated receptors," Circulation, vol. 101, no. 20, pp. 2411-2417, 2000.

[12] L. Teboul, M. Febbraio, D. Gaillard, E. Z. Amri, R. Silverstein, and P. A. Grimaldi, "Structural and functional characterization of the mouse fatty acid translocase promoter: activation during adipose differentiation," Biochemical Journal, vol. 360, no. 2, pp. 305-312, 2001.

[13] H. Vosper, L. Patel, T. L. Graham et al., "The peroxisome proliferator-activated receptor delta promotes lipid accumulation in human macrophages," Journal of Biological Chemistry, vol. 276, no. 47, pp. 44258-44265, 2001.

[14] A. Chawla, C. H. Lee, Y. Barak et al., "PPARdelta is a very lowdensity lipoprotein sensor in macrophages," Proceedings of the National Academy of Sciences of the United States of America, vol. 100, no. 3, pp. 1268-1273, 2003.

[15] M. C. Jong, M. J. Gijbels, V. E. Dahlmans et al., "Hyperlipidemia and cutaneous abnormalities in transgenic mice overexpressing human apolipoprotein C1," Journal of Clinical Investigation, vol. 101, no. 1, pp. 145-152, 1998.

[16] S. J. Laurent, M. I. Mednieks, and R. L. Rosenfield, "Growth of sebaceous cells in monolayer culture," In Vitro Cellular \& Developmental Biology, vol. 28A, no. 2, pp. 83-89, 1992.

[17] T. Willson, "Chemical genomics of orphan nuclear receptors," Ernst Schering Research Foundation Workshop, no. 42, pp. 2942, 2003.
[18] J. Berger, M. D. Leibowitz, T. W. Doebber et al., "Novel peroxisome proliferator-activated receptor (PPAR) gamma and PPAR $\delta$ ligands produce distinct biological effects," Journal of Biological Chemistry, vol. 274, no. 10, pp. 6718-6725, 1999.

[19] N. Suh, Y. Wang, C. R. Williams et al., "A new ligand for the peroxisome proliferator-activated receptor- gamma (PPARgamma), GW7845, inhibits rat mammary carcinogenesis," Cancer Research, vol. 59, no. 22, pp. 5671-5673, 1999.

[20] L. M. Leesnitzer, D. J. Parks, R. K. Bledsoe et al., "Functional consequences of cysteine modification in the ligand binding sites of peroxisome proliferator activated receptors by GW9662," Biochemistry, vol. 41, no. 21, pp. 6640-6650, 2002.

[21] R. L. Rosenfield, "Relationship of sebaceous cell stage to growth in culture," Journal of Investigative Dermatology, vol. 92, no. 5, pp. 751-754, 1989.

[22] K. N. Qin, M. I. New, and K. C. Cheng, "Molecular cloning of multiple cDNAs encoding human enzymes structurally related to $3 \alpha$-hydroxysteroid dehydrogenase," Journal of Steroid Biochemistry and Molecular Biology, vol. 46, no. 6, pp. 673-679, 1993.

[23] R. J. Havel and J. P. Kane, "Introduction: structure and metabolism of plasma lipoproteins," in The Metabolic \& Molecular Bases of Inherited Disease, C. R. Scriver, A. L. Beaudet, W. S. Sly, and D. Valle, Eds., pp. 2705-2716, McGrawHill, New York, NY, USA, 8th edition, 2001.

[24] T. L. Innerarity, R. E. Pitas, and R. W. Mahley, "Disparities in the interaction of rat and human lipoproteins with cultured rat fibroblasts and smooth muscle cells. Requirements for homology for receptor binding activity," Journal of Biological Chemistry, vol. 255, no. 23, pp. 11163-11172, 1980.

[25] M. J. Kim, D. Deplewski, N. Ciletti, S. Michel, U. Reichert, and R. L. Rosenfield, "Limited cooperation between peroxisome proliferator-activated receptors and retinoid X receptor agonists in sebocyte growth and development," Molecular Genetics and Metabolism, vol. 74, no. 3, pp. 362-369, 2001.

[26] C. Bastie, D. Holst, D. Gaillard, C. Jehl-Pietri, and P. A. Grimaldi, "Expression of peroxisome proliferator-activated receptor PPAR $\delta$ promotes induction of PPAR $\gamma$ and adipocyte differentiation in ${ }_{3} \mathrm{~T}_{3} \mathrm{C} 2$ fibroblasts," Journal of Biological Chemistry, vol. 274, no. 31, pp. 21920-21925, 1999.

[27] W. R. Oliver Jr., J. L. Shenk, M. R. Snaith et al., "A selective peroxisome proliferator-activated receptor delta agonist promotes reverse cholesterol transport," Proceedings of the National Academy of Sciences of the United States of America, vol. 98, no. 9, pp. 5306-5311, 2001.

[28] A. Chawla, W. A. Boisvert, C. H. Lee et al., "A PPAR gamma-LXR-ABCA1 pathway in macrophages is involved in cholesterol efflux and atherogenesis," Molecular Cell, vol. 7, no. 1, pp. 161-171, 2001.

[29] Y. X. Wang, C. H. Lee, S. Tiep et al., "Peroxisome-proliferatoractivated receptor delta activates fat metabolism to prevent obesity," Cell, vol. 113, no. 2, pp. 159-170, 2003.

[30] N. Abumrad, C. Harmon, and A. Ibrahimi, "Membrane transport of long-chain fatty acids: evidence for a facilitated process," Journal of Lipid Research, vol. 39, no. 12, pp. 23092318, 1998.

[31] M. Febbraio, N. A. Abumrad, D. P. Hajjar et al., "A null mutation in murine CD36 reveals an important role in fatty acid and lipoprotein metabolism," Journal of Biological Chemistry, vol. 274, no. 27, pp. 19055-19062, 1999.

[32] D. Calvo, D. Gomez-Coronado, Y. Suarez, M. A. Lasuncion, and M. A. Vega, "Human CD36 is a high affinity receptor for the native lipoproteins HDL, LDL, and VLDL," Journal of Lipid Research, vol. 39, no. 4, pp. 777-788, 1998. 
[33] T. Nakatsuji, M. C. Kao, L. Zhang, C. C. Zouboulis, R. L. Gallo, and C. M. Huang, "Sebum free fatty acids enhance the innate immune defense of human sebocytes by upregulating betadefensin-2 expression," Journal of Investigative Dermatology, vol. 130, no. 4, pp. 985-994, 2010.

[34] K. Cianflone, D. A. Roncari, M. Maslowska, A. Baldo, J. Forden, and A. D. Sniderman, "Adipsin/acylation stimulating protein system in human adipocytes: regulation of triacylglycerol synthesis," Biochemistry, vol. 33, no. 32, pp. 9489-9495, 1994.

[35] N. R. Coe and D. A. Bernlohr, "Physiological properties and functions of intracellular fatty acid- binding proteins," Biochimica et Biophysica Acta, vol. 1391, no. 3, pp. 287-306, 1998.

[36] L. Makowski, J. B. Boord, K. Maeda et al., "Lack of macrophage fatty-acid-binding protein aP2 protects mice deficient in apolipoprotein E against atherosclerosis," Nature Medicine, vol. 7, no. 6, pp. 699-705, 2001.

[37] D. Thiboutot, A. Sivarajah, K. Gilliland, Z. Cong, and G. Clawson, "The melanocortin 5 receptor is expressed in human sebaceous glands and rat preputial cells," Journal of Investigative Dermatology, vol. 115, no. 4, pp. 614-619, 2000.

[38] L. Zhang, W. H. Li, M. Anthonavage, and M. Eisinger, "Melanocortin-5 receptor: a marker of human sebocyte differentiation," Peptides, vol. 27, no. 2, pp. 413-420, 2006.

[39] A. J. Thody and S. Shuster, "Control of sebaceous gland function in the rat by alpha-melanocyte stimulating hormone," Journal of Endocrinology, vol. 64, no. 3, pp. 503-510, 1975.

[40] A. J. Thody, M. F. Cooper, P. E. Bowden, D. Meddis, and S. Shuster, "Effect of alpha-melanocyte stimulating hormone and testosterone on cutaneous and modified sebaceous glands in the rat," Journal of Endocrinology, vol. 71, no. 3, pp. 279-288, 1976.

[41] W. Chen, M. A. Kelly, X. Opitz-Araya, R. E. Thomas, M. J. Low, and R. D. Cone, "Exocrine gland dysfunction in MC5-Rdeficient mice: evidence for coordinated regulation of exocrine gland function by melanocortin peptides," Cell, vol. 91, no. 6, pp. 789-798, 1997.

[42] M. Rosenbaum and R. L. Leibel, "The role of leptin in human physiology," New England Journal of Medicine, vol. 341, no. 12, pp. 913-915, 1999.

[43] M. Y. Wang, Y. Lee, and R. H. Unger, "Novel form of lipolysis induced by leptin," Journal of Biological Chemistry, vol. 274, no. 25, pp. 17541-17544, 1999.

[44] H. Yagyu, T. Kitamine, J. Osuga et al., "Absence of ACAT-1 attenuates atherosclerosis but causes dry eye and cutaneous xanthomatosis mice with congenital hyperlipidemia," Journal of Biological Chemistry, vol. 275, no. 28, pp. 21324-21330, 2000.

[45] J. F. Reindel, M. A. Dominick, T. M. Bocan, A. W. Gough, and E. J. McGuire, "Toxicologic effects of a novel acyl-CoA: cholesterol acyltransferase inhibitor in cynomolgus monkeys," Toxicologic Pathology, vol. 22, no. 5, pp. 510-518, 1994.

[46] H. C. Chen, S. J. Smith, B. Tow, P. M. Elias, and R. V. Farese Jr., "Leptin modulates the effects of acyl CoA: diacylglycerol acyltransferase deficiency on murine fur and sebaceous glands," Journal of Clinical Investigation, vol. 109, no. 2, pp. 175-181, 2002.

[47] Y. Zheng, K. J. Eilertsen, L. Ge et al., "Scd1 is expressed in sebaceous glands and is disrupted in the asebia mouse," Nature Genetics, vol. 23, no. 3, pp. 268-270, 1999.

[48] Y. Zheng, S. M. Prouty, A. Harmon, J. P. Sundberg, K. S. Stenn, and S. Parimoo, "Scd3 -a novel gene of the stearoyl-CoA desaturase family with restricted expression in skin," Genomics, vol. 71, no. 2, pp. 182-191, 2001.

[49] R. L. Rosenfield, D. Deplewski, A. Kentsis, and N. Ciletti, "Mechanisms of androgen induction of sebocyte differentiation,” Dermatology, vol. 196, no. 1, pp. 43-46, 1998. 


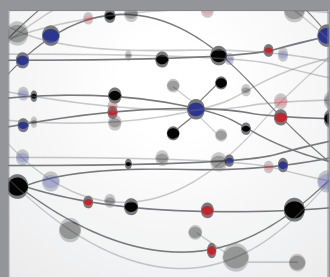

The Scientific World Journal
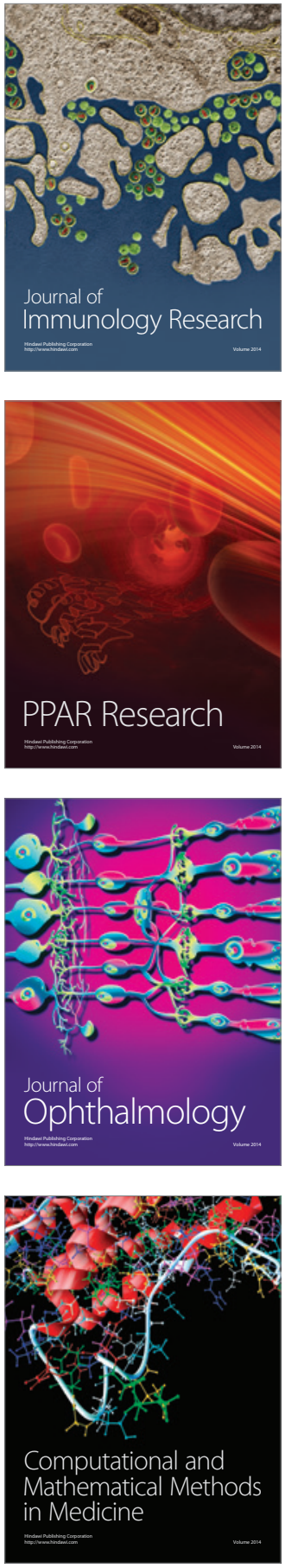

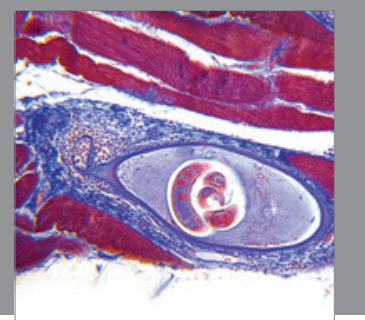

Gastroenterology

Research and Practice
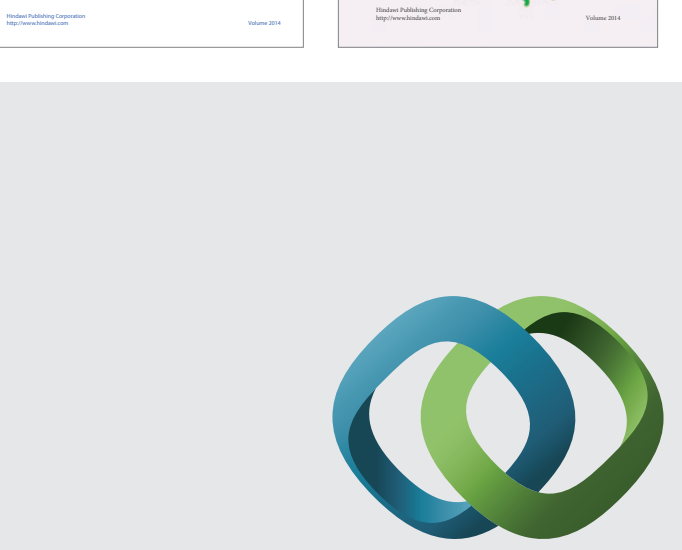

\section{Hindawi}

Submit your manuscripts at

http://www.hindawi.com
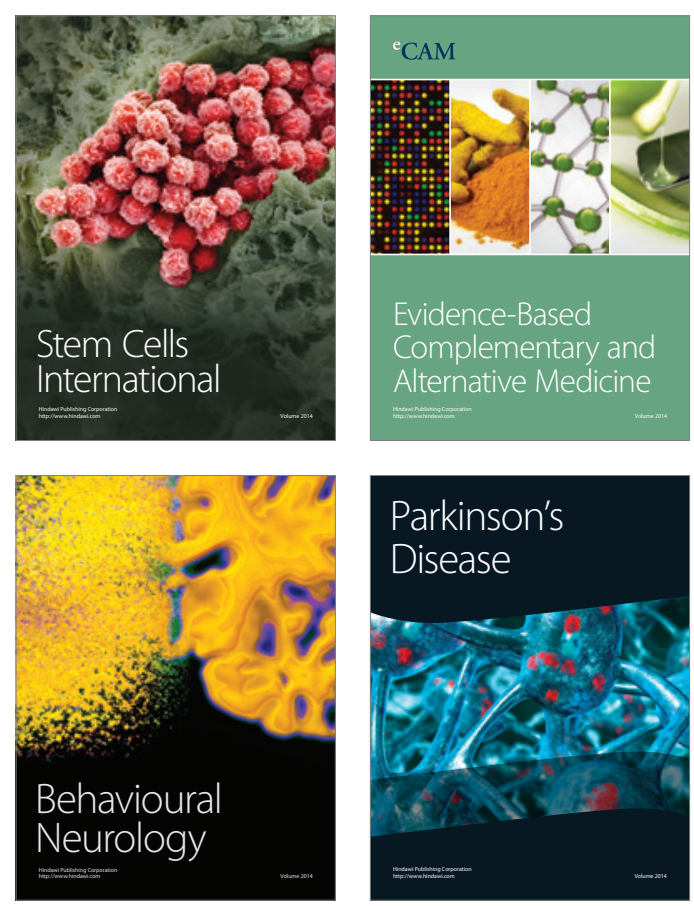

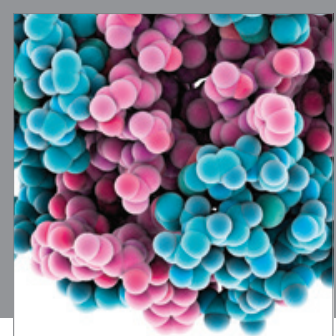

Journal of
Diabetes Research

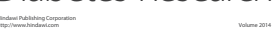

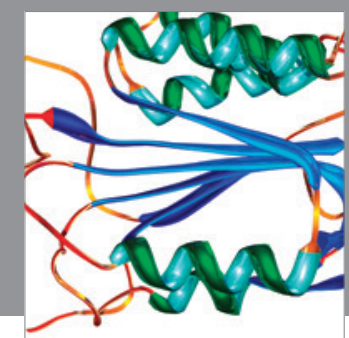

Disease Markers
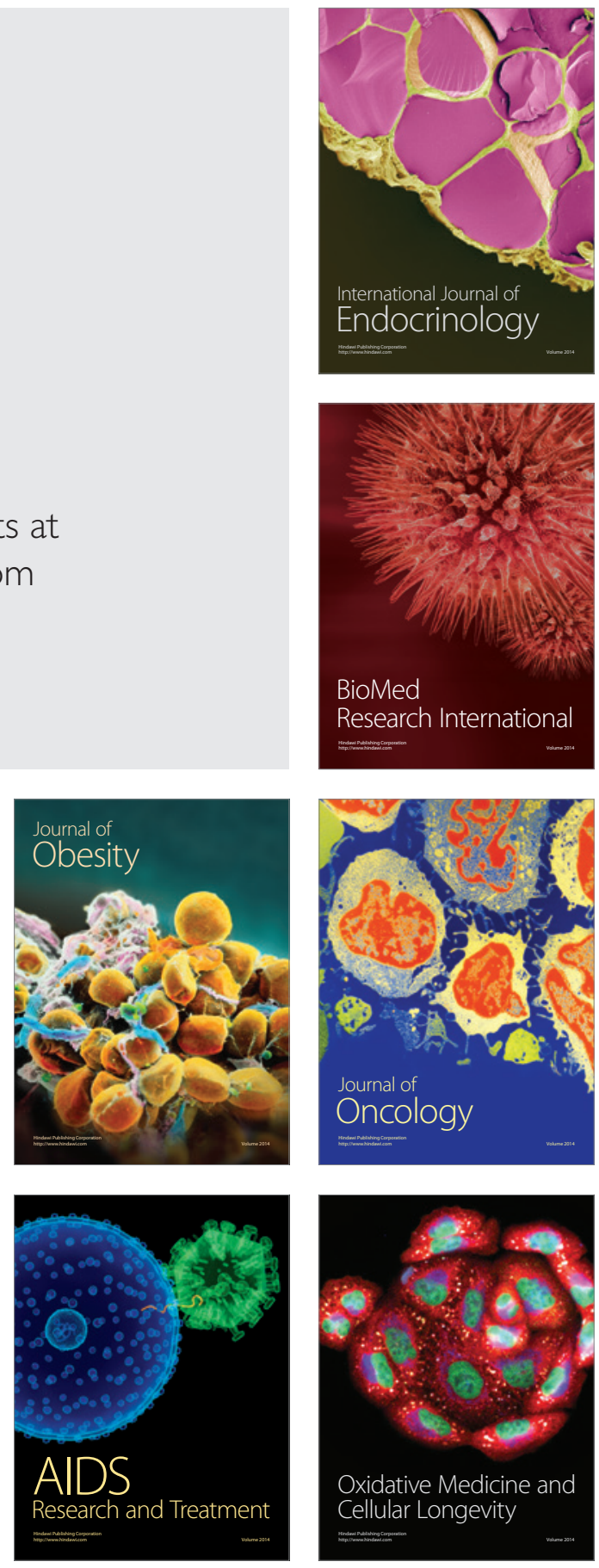